Military Technical College

Kobry El-Kobba

Cairo, Egypt

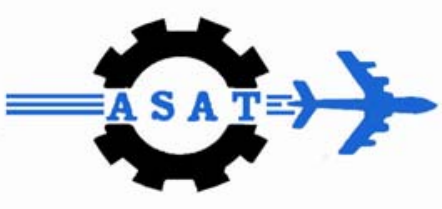

12-th International Conference

on

Aerospace Sciences \&

Aviation Technology

\title{
A MODELING OF NEGATIVE RESISTANCE MOSFET FOR METAMATERIALS LOW LOSS OSCILLATORS FOR NANOTECHNOLOGY APPLICATIONS
}

\author{
MOHAMED ALAZAB
}

\begin{abstract}
This paper presents analysis of the Metamaterials parameters and its dependence on its nanostructure and have linear electric permittivity and magnetic permeability. These parameters affect functionally the energy propagation and losses. It also investigates that these materials need to employ a controllable negative resistance to control the above mentioned parameters and compensate for the nanometric oscillator losses. A new nonnumeric negative resistance MOSFET ( NR-MOSFET) has been proposed, designed and examined for this reason. These losses results from metallic sub wavelength Split-Ring Resonator (SRR) and the metamaterial itself is constructed of arrays of these SRR cells. The basic idea of the negative resistance MOSFET depends on contracting the channel width proportionally as increasing the drain voltage. The new device is expected to have a speed of response which is higher than any of the known traditional techniques, a very low power consumption and a wide dynamic range of resistance variation. This good performance serve to have the desired functionality of the metamaterial.
\end{abstract}




\section{INTRODUCTION}

Metamaterials are nanostructure materials fabricated from repetition of dielectric and I or metallic elements to produce the desired electromagnetic behavior and they are characterized by negative electric permittivity $-\varepsilon$ and magnetic permeability $-\mu$ which makes it to have negative refractive index $n$ and energy propagating in the opposite direction of the phase velocity [1-2]as shown in Figure (1) .

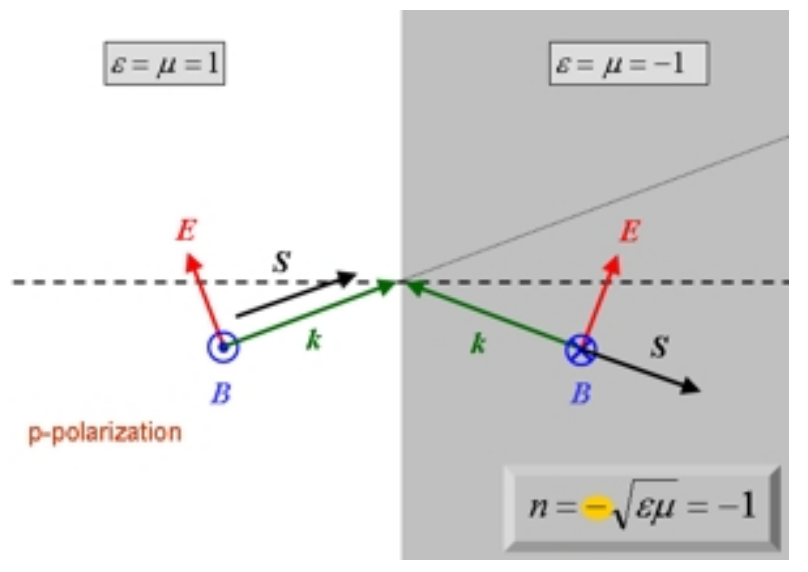

Figure (1) Negative refraction at the interface between vacuum and metamaterial .

The idea of metamaterials is based on an array of metallic sub-wavelength split-ring resonator (SRR). Each of the SRR mimics an LC-Oscillator consisting of a magnetic coil with inductance $L$ and a capacitor with capacitance $C$ as shown in Figure (2) .

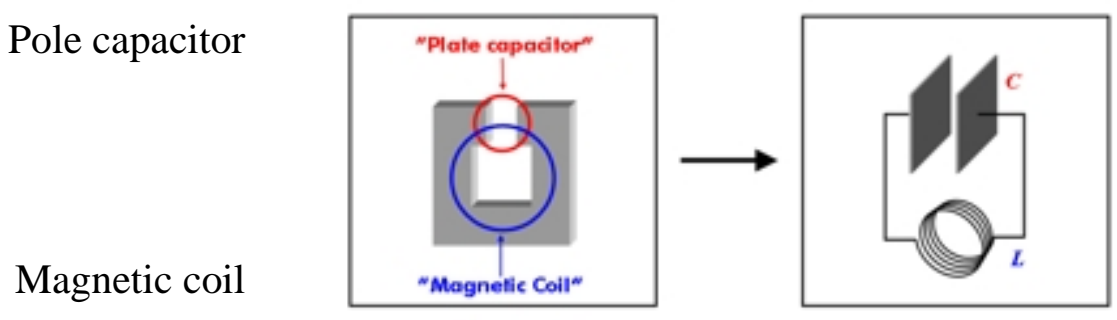

Figure (2). The basic physics of a SSR simulated as an LC oscillator

If the frequency of the incident light lies within a spectral band slightly above the LCresonance frequency, the effective magnetic permeability of the SRR array can indeed be negative. These cells act as electric dipoles whose electric response can lead to a negative permittivity. As is well known, in order to obtain a magnetic response from a metal nanostructure the incident light has to excite local currents circulating in loops (solenoid currents). These currents in turn give rise to a magnetic dipole-moment. By properly designing the metal nanostructure, one can obtain a resonant enhancement of 
the local currents leading to strong magnetic response, and if a current is excited and circulated in monomolecular loops, it gives rise to a magnetic dipole moment, which in turn gives a current which circulates in the same direction as that creating the magnetic dipole moment. This latter current enhances by the same manner the magnetic dipole moment and so on, leading to strong magnetic response with negative permeability and does the same w.r.t. the electric response and gives rise to negative permittivity [3]. As shown in Figure (3), I' meta assists I and B' meta assists B.

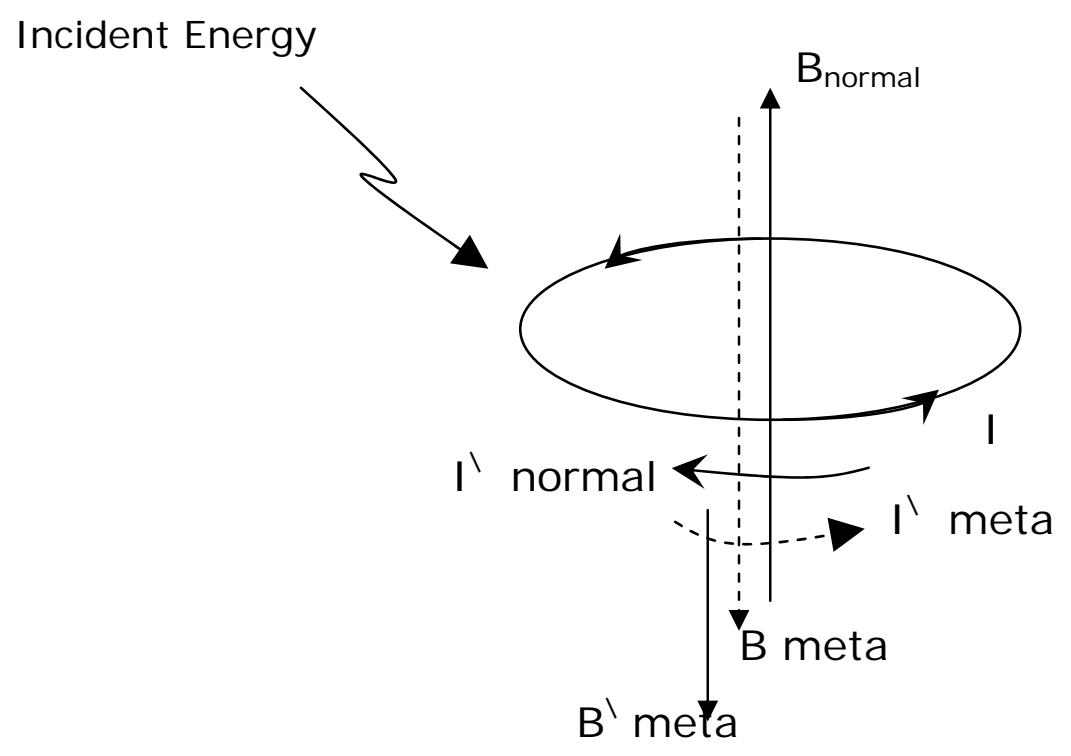

Figure (3) The circulated currents and the magnetic dipole moments.

This property is very needed in many applications such as antenna where the radiation is noticeably increased and Several advantages of such nanostructure materials are gained. Size reeducation, high directivity, increased power radiability and novel functionalities are examples of these advantages.

This meatmaterial oscillator has a power loss due to the series resistance of the coil. Work presented by $\mathrm{M}$. Abdalla et al [ 4] for compensating the resistance with an active negative CMOS resistance and the idea is to reduce the losses not to eliminate it as the limitations of the Kramers-Krongig relations for lossless particles is considerd in this paper [5]. Our model for negative resistance has advantages of frequency dependent which is compatible with metamaterial, where the metamaterials are dispersive. Hence the positive resistance is function of frequency. Therefore our model to compensate this positive dependent frequency resistance is also frequency dependent and almost the same functional form [4].

Section II illustrates the Electric / magnetic response enhancement, negative resistance MOSFET ( NR-MOSFET) is presented in section III, theory and modeling in section IV , and measurement technique is presented in section $\mathrm{V}$. The conclusions of this work are given in section 6 . 


\section{ii. ELECTRIC/MAGNETIC RESPOSE ENHANCEMENT}

Knowing that an array of metmaterial SRR can be modeled or equivalent to a series of array of LC sections ( see figure (4)), then the enhancement of the electric and magnetic response can be further enhanced by decreasing the nanostructure resonator losses, which can be fulfilled by inserting a nano structure negative resistance element such as a nanometer negative resistance MOSFET(NR-MOSFET) in series with each array element of SRR [6] .

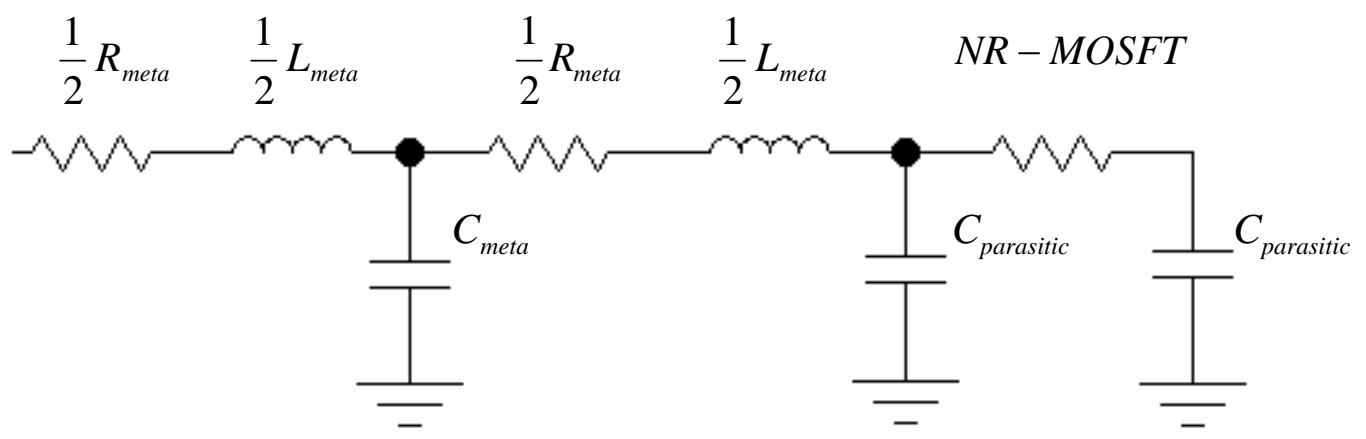

Figure (4) The proposed equivalent circuit for an array of SRR and compensated negative resistance

Where $\frac{1}{2} R_{\text {meta }}, \quad \frac{1}{2} L_{\text {meta }}$ and $C_{\text {meta }}$ are metamaterial equivalent circuit and $N R-M O S F T, C_{\text {parasitic }}$ are the negative resistance compensation.

\section{NEGATIVE RESISTANCE MOSFET}

The idea and construction of the proposed voltage controlled negative resistance MOSFET ( NR-MOSFET) is shown in figure (5). It is realized using the polysilicon gate $\mathrm{N}$-channel MOSFET technology. It consists of a MOSFET $\left(T_{N R}\right)$ with channel length $L_{N}$, width $Z_{N}$ and oxide thickeners $h_{O N}$, the channel width contraction is achieved using two additional $\mathrm{P}^{+}$lateral diffusions whose potential is maintained negative with respect to the channel well potential and its amplitude kept varying proportionally with the drain voltage $V_{\text {Ds.. }}$ To carry out this requirements a MOSFET operational amplifier are designed so as to perform the desired amount of channel contraction. This operational amplifier is biased between $+V_{R}$ and $-V_{R}$ with $V_{R}$ being the control voltage [7-8 ]. 


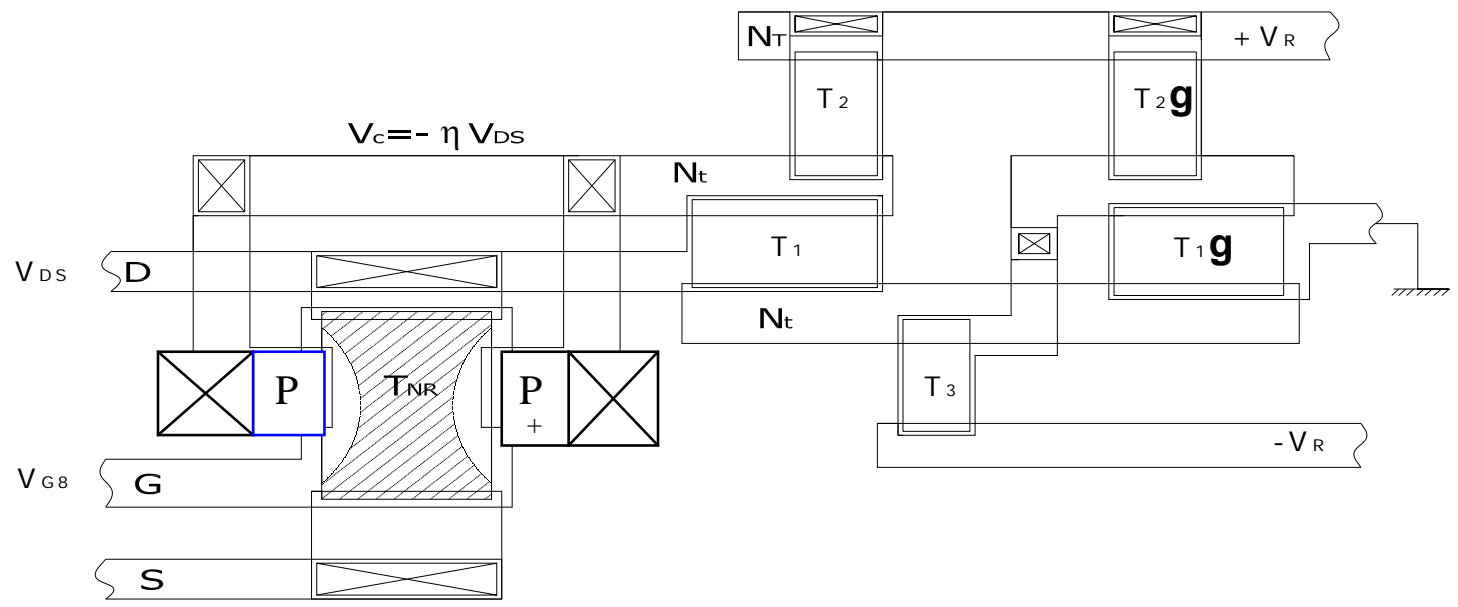

Figure (5) The construction of the proposed voltage controlled negative resistance

A MOSFET operational amplifier is designed so as to reverse the polarity and have the desired amplitude needed to elaborate the wanted contraction and it is biased between the control voltages $+V_{R},-V_{R}$. The equivalent circuit of the controlled negative resistance is shown in Figure (6).

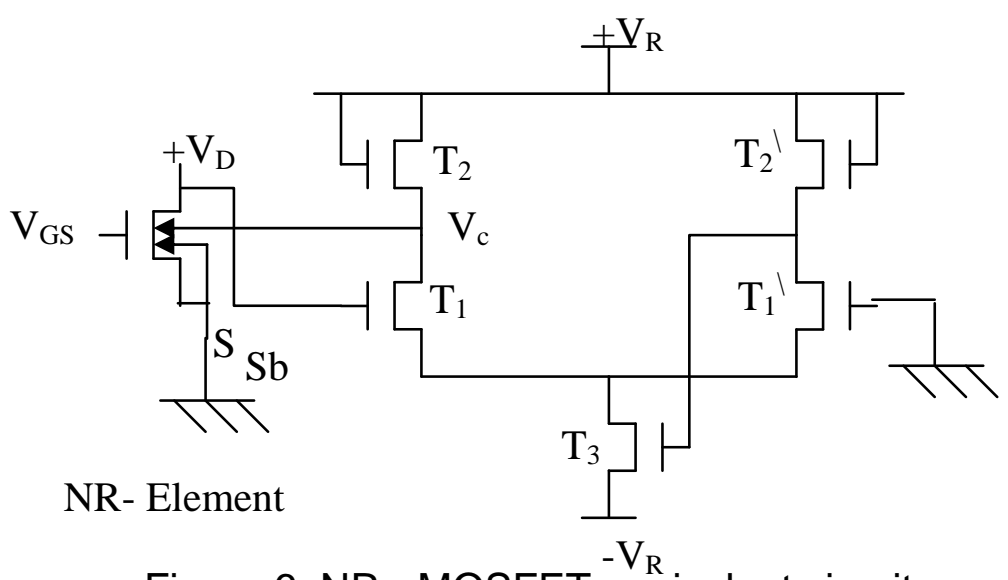

Figure 6. NR - MOSFET equivalent circuit

The op amp is designed so as the control voltage $V_{C}$ obeys the behavior shown in figure (7), and goes negative as $V_{D S}$ goes positive. Since the channel well potential is always negative this design guarantees a strong depletion of the $N^{+}$version layer of the channel well. This leads to a depletion layer width $W$ which increases steeply as $V_{D S}$ increases, thus controlled decrease of the channel width and consequently channel current $I_{D S}$ decreases as $V_{D S}$ increases; we notice that the decrease of $I_{D S}$ becomes more noticeable in the saturation region where $I_{D S}$ should remain constant and cause more observable negative resistance behavior. 


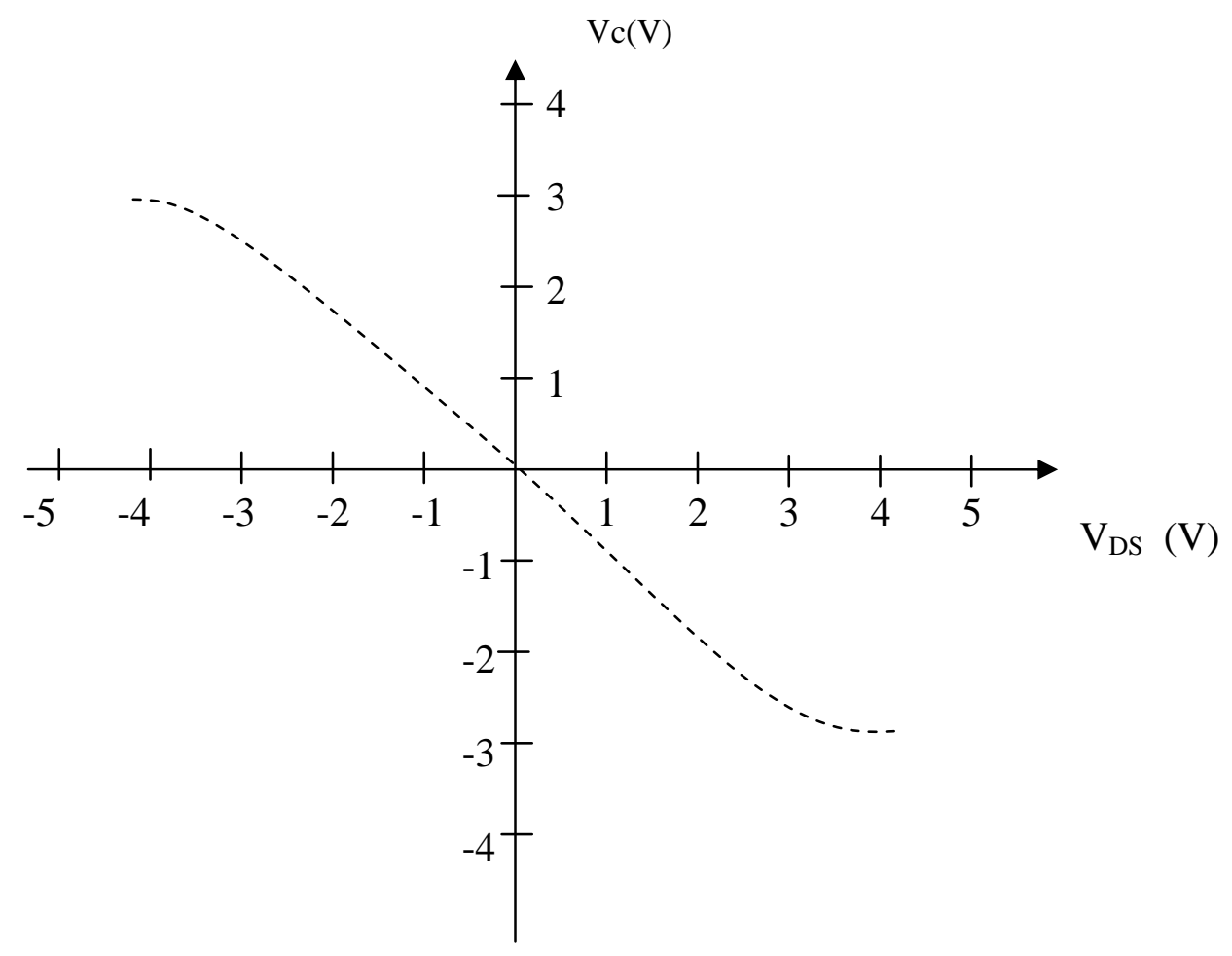

Fig.(7) Variation of $V_{c}$ with $V_{D S}$ ( the working are is in the linear region only from $+3 \mathrm{~V}$ to $-3 \mathrm{~V}$ )

\section{THEORY AND MODELING}

In this section we evaluate and model the depletion layer width, the effective channel width and the frequency and voltage controlled negatve resistance.

\section{A. EVALUATION OF THE CHANNEL CONTRACTION DUE TO DRAIN AND GATE VOLTAGES}

The variation of the channel width $W$ and its dependence on the drain and gate voltages in saturation region. Referring to figure ( 8 ) which shows the energy band diagram of the proposed negative resistance NR-MOSFET[10-12]. 


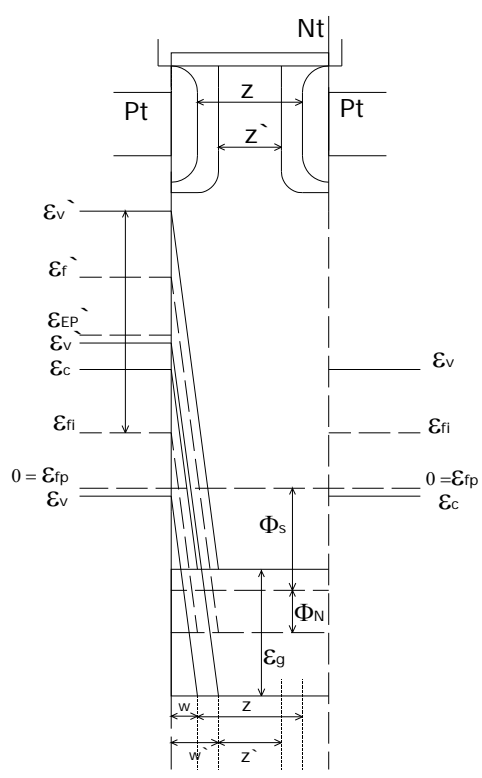

Figure 8. the energy band diagram for NR - MOSFET

The variation of the potential $V_{Z}$ with distance, when the Al Langue potential is made zero is given by:

$$
V_{Z}=V_{C}+\frac{C_{o x}}{2 d_{o} \varepsilon_{s}}\left(V_{G S}-V_{T}-V_{x}\right) Z^{2}
$$

where $V_{c}$ is the control voltage

$$
V_{c}=-\eta V_{D S}, \quad \eta=\frac{\sqrt{Z_{1} / L_{1}}}{\sqrt{Z_{2} / L_{2}}}
$$

$Z$ is the channel width, and $Z_{1}, Z_{2}, L_{1}$ and $L_{2}$ are the channel width and length of opamp MOSFET $T_{1}$ and $T_{2}$

$d_{o}$ the MOSFET channel depth $\left(\sim 100 A^{\circ}\right)$, $\mathrm{C}_{\mathrm{ox}}$ is the oxide thickness $V_{T}$ the threshold voltage $(\sim 0.5 \mathrm{~V})$, $V_{x}$ the channel potential taken, longitudinally, at postion $X(V)$.

The amount of channel contraction can be calculated by equating the channel voltage to the surface potential, one can find: 


$$
W=\sqrt{\frac{2 d_{0} \varepsilon_{S}\left(V_{S}+\eta V_{D S}\right)}{C_{o x}\left(V_{G S}-V_{T}-V_{X}\right)}}
$$

The effective channel width can be expressed in terms of position $X$ in the channel from the source and the biasing voltages $V_{D S}$ and $V_{G S}$ as follows:

$$
Z_{\text {effective }}=Z-2 W
$$

\section{B. NEGATIVE RESISTANCE MOSFET}

The modified drain current equation in the saturation region is therefore:

$$
I_{D S}=\beta \frac{1}{2}\left[V_{G S}-V_{T}-V_{D S} \frac{X}{L}\right]^{2}
$$

where $\beta=\mu C_{\text {ox }} \frac{Z_{\text {effective }}}{L}$,

The Resistance equivalent

$$
R_{N}=\frac{d V_{D S}}{d I_{D S}}
$$

$$
R_{N}=-\frac{L^{2}}{\mu C_{o x}(Z-2 W)\left(V_{G S}-V_{T}-V_{D S} \frac{X}{L}\right)}
$$

This indicates that the NR-MOSFET acquire a negative resistance in saturation region of operation. Controlling the geometrical ratio of the NR controls this resistance.

To realize a nano geometric NR-MOSFET advanced fabrication process based on ion implantation will be used instead of the traditionally known photolithographic technique to implement all drain, source and gate regions [ 9-10]. 
We can see as the negative resistance can be controlled by the frequency and the contraction of the channel width until we reach to the low loss of the metamatrials cell.

\section{MEASUREMENT TECHNIQUE}

Experimental test MOSFETs have been prepared by Thomson microelectronics DRD of Grenoble / France. The source channel-width $Z=10$ micron, the drain channel-width $Z$ ranges from 5 to 10 micron, channel length $L=5$ micron and oxide thickness $h_{0}$ are 40 $A^{\circ}$ and $1200 A^{\circ}$. Measurements are performed on these test specimens to obtain the value of the effective MOSFET geometrical ratio $\left(\frac{Z}{L}\right)_{\text {eff }}$ and its dependence on the device geometry $(Z, L, \beta)$ and biasing $\left(V_{D S}, V_{G S}\right)$. Measurement and characterization are achieved using the experiential set up shown in Figure 9. Hundreds of measurements have been performed to specify the device parameters .

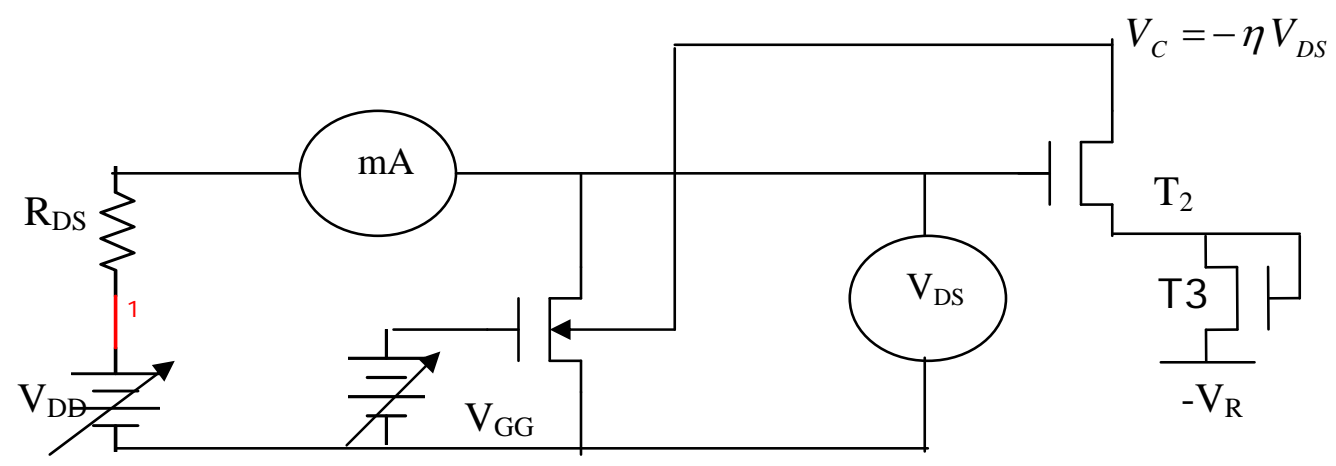

Figure 9. Experimental Setup.

Figure (10) shows the dependence of the $I_{D S}-V_{D S}$ characteristics on the value of the parameter $\eta$ and geometries. We observe that $I_{D S}$ still increasing proportionally in the ohmic region with $V_{D S}$ but at smaller rate. Then $I_{D S}$ begins to decrease as increasing $V_{D s}$ in the saturation region. The rate of decreases becomes more important at greater 
values of $\eta$. This behavior is attributed to the increase of the depletion region width with $V_{D S}$. It becomes more noticeable of at greater values of $V_{G S}$.

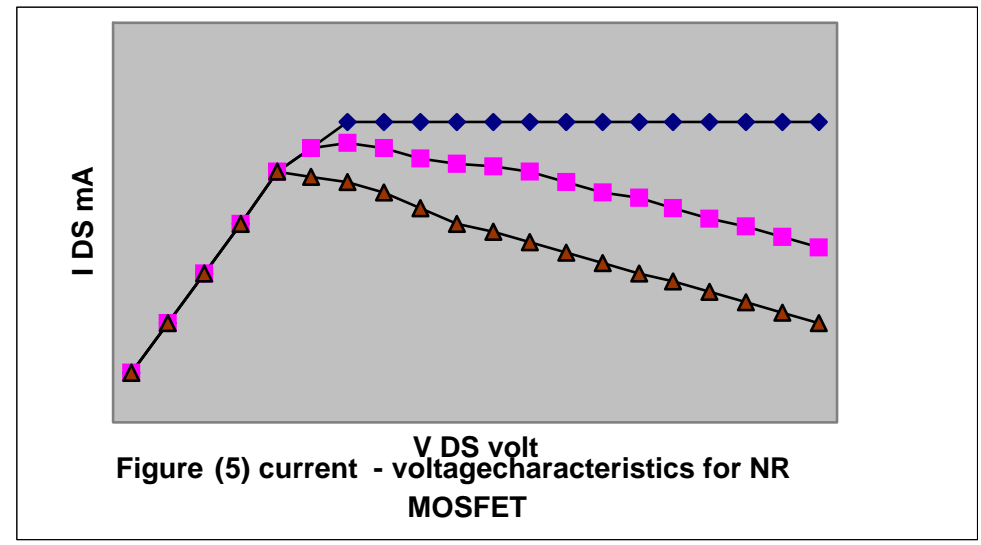

Figure 10. Current -Voltage characteristics for NR-MOSFET

\section{CONCLUSIONS}

This paper gives a simple analytical model for frequency dependent, voltage controlled, low loss oscillator metamterial cell which is composed of SRR cell and a nanometer negative resistance NR-MOSFET. Although the cell always remains causal, the limitations like the Kramers-Kronig relation (for lossless particles) can be overcome in this design. This is achieved where a power generated can be realized by active loads which clearly violates the Kramers-Kronig relation, Which required besides causality the system be linear and memory effects decay in time [11-12].

We can finally conclude that the paper present a general approach to the design of an effective class of metamaterials. We hope that the model of this paper clearly demonstrate the power of the metamaterial concept that allows a great flexibility in the design and applications of nanotechnology .

\section{ACKNOWLEDGMENT}

The author wish to thank Professor ADEL EL-HENAWE for the opportunity to present these ideas and clarifying various points. 


\section{REFERENCES}

[1] R. A. Shelby, n. Smith and S. Schultz, "Experimental verification of a negative index of refraction", Science, vol. 229, pp.77-79, 2001.

[2] N. Engheta, "An idea for thin subwavelength cavity resonators using metmaterials with negative permittivity and permeability", IEEE antennas and wireless propagation letters, vol. 1, no. 1, pp.10-13, 2002.

[3] P. Markos and C. M. Soukoulis, Phys. Rev. E 65, 036622(2002).

[4] M. Abdalla et al"A differential 0.13uM CMOS active Inductor for high-frequency shifters", IEEE ISCAS 2006, pp. 3341-3344 .

[5] Mark I. Stockman, " Does nature allow negative refraction with low losses in optical region?", arXiv:cond-mat/0611350 v! 14 Nov 2006.

[6] Vainikainen. $P$ et al "Advances in Diversity performance Analysis of Mobile Terminal Antennas" International symposium on Antennas and propagation ISAP 2004, Sendai,Japan, August 2004, pp.649-652

[7] A. El-Hennawy E. Al-MAZUKi , and S.A.L Ghamdi , "Modeling and characterization of a new negative resistance NR -MOSFET for VLSI application. ICM 91,Proc. the International Conference. Micro electronics, Cairo, Egypt December 1991.

[8] S.Msze , physics of semi conductor Devices thon , wiley\& sons NY, 1984

[9] Chua, L. Juebang Yu Youying Yu "Bipolar - JFET - MOSFET negative resistance devices", Circuits and Systems, IEEE Transactions on, Publication Date: Jan 1985, Volume: 32, Issue: 1, PP:46- 61, ISSN: 0098-4094.

[10] A. RAMADAN, A. EL-HENNAY, HASSAN K., ALI ABOU EL-NOUR " Study and characterization of a new MOSFET voltage controlled negative resistance for super selective IC tank circuits", 1999, vol. 86, n⿳30, pp. 311-319 (9 ref.), International journal of electronics (Int. j. electron.) ISSN 0020-7217

[ 11] Ciais ,P., taraj, R., Kossiavas, G. and Luxey, C Design of an Internal Quad-Band Antenna for Mobile phones", IEEE Microwave and Wireless Components Letters, vo1.14 no. 4 April 2004, pp.148-150.

[12] Lee, T. H., The design of CMOS Radio-Frequency Integrated Circuits, Chapt. 11, Cambridge U. Press, 1998. 